\title{
Therapy resistant traumatic fungal infection necessitating arm amputation after free flap coverage
}

\author{
A. Dall'Antonia • M. Hamdi • K. Van Landuyt • \\ Ph. N. Blondeel $\cdot$ N. Roche $\cdot$ S. J. Monstrey
}

Received: 13 February 2007 / Accepted: 27 February 2008 / Published online: 8 July 2008

(C) Springer-Verlag 2008

\begin{abstract}
The indication for microvascular free tissue transfer is usually exposure of vital structures, bone defect, or loss of function. Radical debridement and control of infection is a prerequisite for any kind of reconstructive procedure. This is especially true for untidy wounds in industrial or agricultural settings; in these, one should not hesitate to debride any tissue which is not obviously viable. Aggressive surgical debridement should be performed before flap coverage. Some organisms, however, fail to be eradicated by mechanical debridement as they are particularly pathogenic or embedded in the tissues. Perforator flaps are currently recommended for soft tissue coverage of the upper limb, for their pliability and the low morbidity of the donor site. A case of traumatic deglovement injury of an upper arm is presented. After free flap coverage, it was complicated by a rare and highly pathogenic fungal infection. Successful soft tissue reconstruction obtained with a chimeric free flap was compromised by an expanding fungal infection ultimately leading to limb amputation.
\end{abstract}

Keywords Upper limbs · Mucormycosis · Perforator flap · Complications

Presented at the Annual Winter Meeting of the Italian Society of Plastic and Reconstructive Surgery, Cortina d'Ampezzo (B1), 26-31 Jan 2004.

A. Dall'Antonia $\cdot$ M. Hamdi $\cdot$ K. Van Landuyt $\cdot$ P. N. Blondeel N. Roche $\cdot$ S. J. Monstrey

Department of Plastic and Reconstructive Surgery,

University Hospital of Ghent,

Ghent, Belgium

A. Dall'Antonia $(\triangle)$

U.O. Chirurgia Plastica, Spedali Civili di Brescia,

Pzz.le Spedali Civili, 1, Brescia 25125, Italy

e-mail: ale.dallantonia@libero.it

\section{Introduction}

A large defect can be defined as any defect which cannot be closed primarily or by simple random local flaps [6]. Microsurgical free tissue transfer is currently the gold standard for coverage and limb salvage of extensive defects of the upper extremity, if they are too large for local flap cover.

The indication for microvascular free tissue transfer is usually exposure of vital structures, bone defect, and loss of function. Surgery may be performed acutely a few days after the trauma or within 2 weeks $[3,10]$.

Early wound cover and infection control, repair of damaged structures, and preservation of limb function are the primary goals [5], but donor site morbidity and contour characteristics are becoming more and more important with the improvement in surgical techniques [6]. Radical debridement and control of infection is a prerequisite for any kind of reconstructive procedure. This is especially true for untidy wounds in industrial or agricultural settings; with these, one should not hesitate to debride any tissue which is not obviously viable.

Mucormycosis, also referred to as phycomycosis or zygomycosis, is a rare but aggressive opportunistic fungal infection, which is now emerging in importance with the increasing prevalence of immunosuppressive conditions and metabolic states. It is caused by a group of ubiquitous saprophytic organisms of the class Zygomycetes and order Mucorales, which comprises nine genera. Species of Rhizopus Mucor and Absidia are most frequently isolated in infected human tissue. Infection can be acquired by inhalation, ingestion, or the deposition of spores in wounds, and the fungi give rise to pathogenic lesions as a result of invasion and growth within the lumen and walls of major blood vessels, with ensuing thromboembolism which can cause 
ischemia and tissue necrosis. There are several clinical variants: rhino-orbital-cerebral, pulmonary, cutaneous, gastrointestinal, and disseminated. The rhino-orbital-cerebral and pulmonary forms present most commonly and, together with disseminated disease, are associated with the highest mortality, ranging from $78 \%$ to $100 \%$. Cutaneous disease comprises less than $10 \%$ of all cases and is associated with the best prognosis, with less than $10 \%$ mortality [13].

The clinical appearance of cutaneous mucormycosis is insufficient for diagnosis because the clinical manifestations generally do not differ from other soft tissue infections. Similar lesions can be produced by infections with Pseudomonas, Aspergillus, Histoplasma, Cryptococcus, Aeromonas hydrophilia, vasculitis, pyoderma gangrenosum, and other necrotizing infections [9]. Tissue biopsy is essential. The appearance of irregularly shaped, broad, nonseptate, twisted, ribbon-like thick-wall fungal hyphae $(10-20 \mu \mathrm{m})$ with right angle branching is classic [9]. A characteristic histological finding of mucormycosis is hyphal invasion of the blood vessels with occlusion, causing a constellation of thrombosis, infarction, ischemia, necrosis, and hemorrhage.

There are no clinical surgical guidelines to treat cutaneous mucormycosis. There are reports of primary cutaneous mucormycosis being treated with a therapy ranging from chemotherapy without surgery to radical approaches including amputation. The combined surgical and medical antifungal chemotherapeutic approach to treatment remains the standard of care $[9,13]$.

The antifungal drug of choice is amphotericin B in the highest tolerable doses, usually $1-1.5 \mathrm{mg} / \mathrm{kg}$ daily. Higher doses of liposomal amphotericin can be used, usually 5$8 \mathrm{mg} / \mathrm{kg}$, and doses as high as $15 \mathrm{mg} / \mathrm{kg}$ daily have also been advocated. Imidazole drugs, such as itraconazole or voriconazole, have also been used. New treatment options are

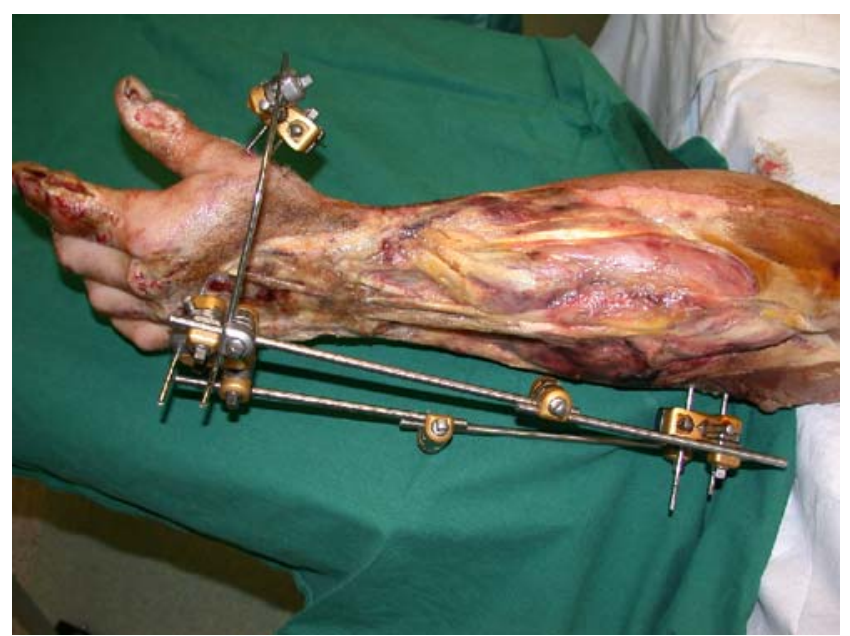

Fig. 1 Preoperative appearance of the defect

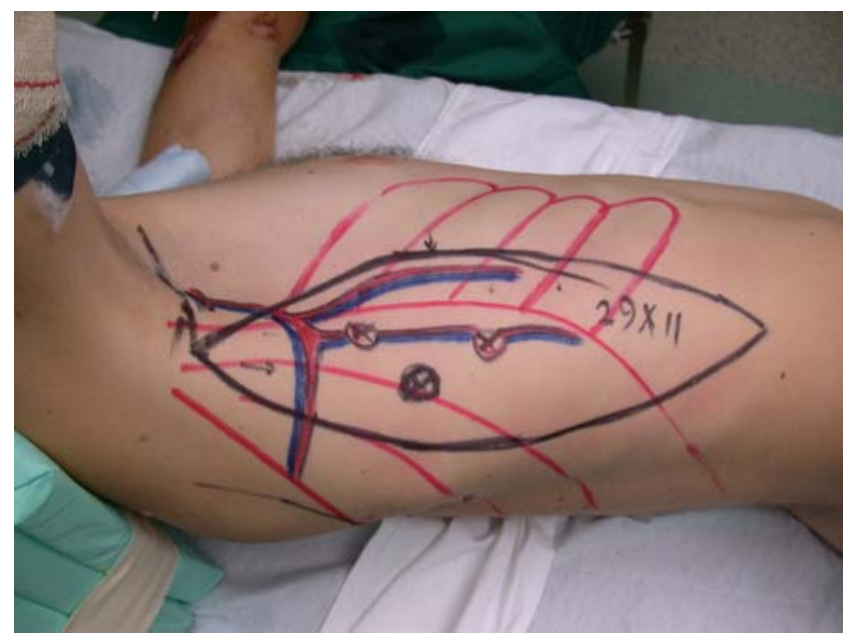

Fig. 2 Preoperative markings of the flap

emerging with triazole antifungal agents such as posaconazole (200 $\mathrm{mg}$ four times daily orally), this is a broad-spectrum antifungal agent which has shown even greater efficacy and less toxicity than amphotericin, and has been suggested as the drug of choice for mucormycosis [9].

\section{Case report}

A 38-year-old farmer was admitted to our hospital after traumatic deglovement of almost his entire arm in a Brussels sprout-harvesting machine. Two transverse fractures of the radius and ulna were reduced and fixed with external fixation. There was also an intra-articular fracture of the radius with carpal subluxation (Fig. 1). Wide debridement of the wound was performed and high dose intravenous antimicrobial therapy was instigated using amoxicillin/clavulanate potassium.

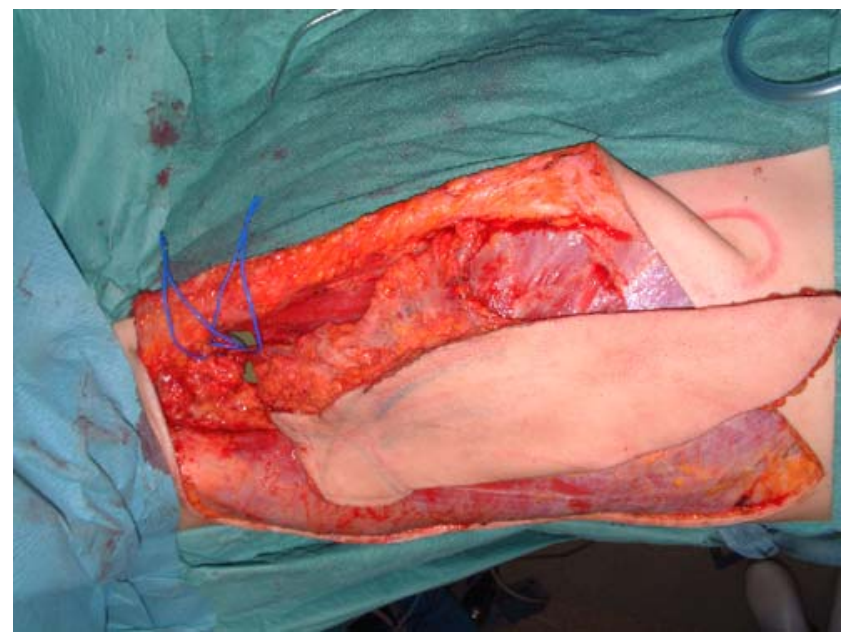

Fig. 3 Intraoperative view of flap harvesting 


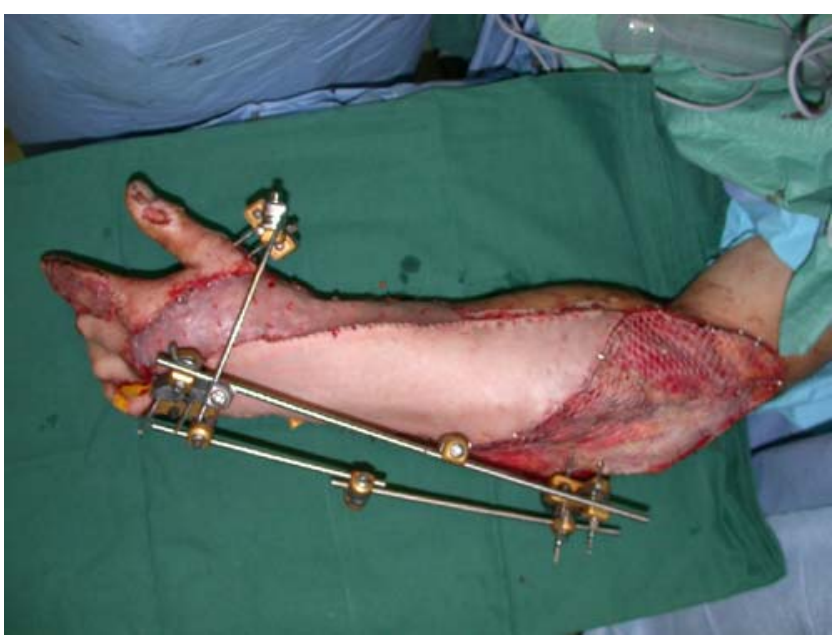

Fig. 4 Immediate postoperative view: full cover of the defect

Six days later the patient underwent debridement of the lesion and the defect was covered with a free "chimera" flap [7] (Fig. 2). This was a thoracodorsal artery flap with a segment of latissimus dorsi muscle to cover such a large defect. Unfortunately, there was no satisfactory perforator found during the dissection and the flap was harvested on the lateralis thoracic artery and its comitant veins [8] (Fig. 3). This flap had a skin paddle together with the serratus fascia which was skin grafted (Fig. 4). The anastomosis of the artery was performed end-to-side to the radial artery and the vein end-to-side to a superficial vein.

Three days after surgery, a fungal infection by Mucormycosis was discovered and was treated immediately with amphotericin B (Fig. 5). The treatment with amphotericin B was suspended after 7 days due to impending renal failure. A specific antimicotic agent targeted against Mucormycosis+, still under investigation for regulatory approval, posaconazole [11] was started to control the fungal spread.

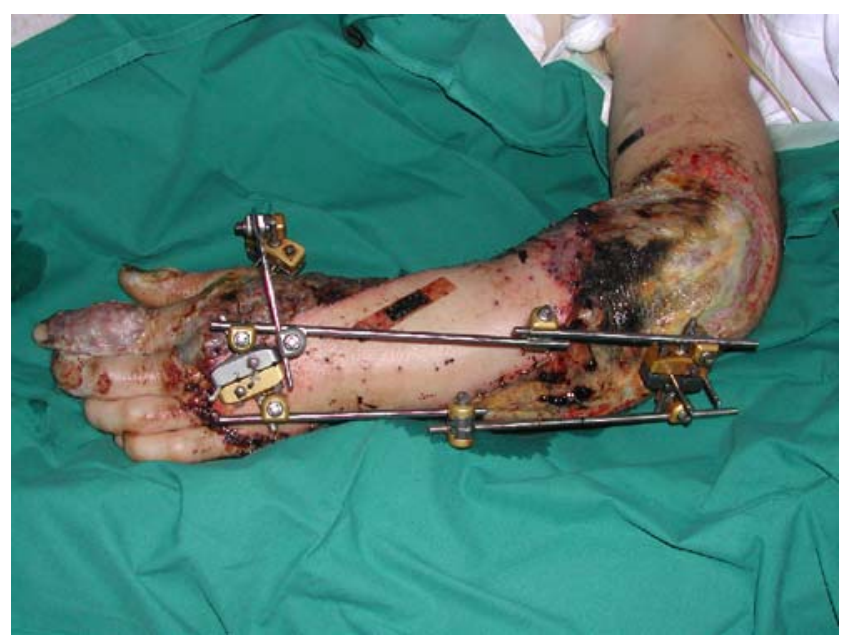

Fig. 5 Persistent infection

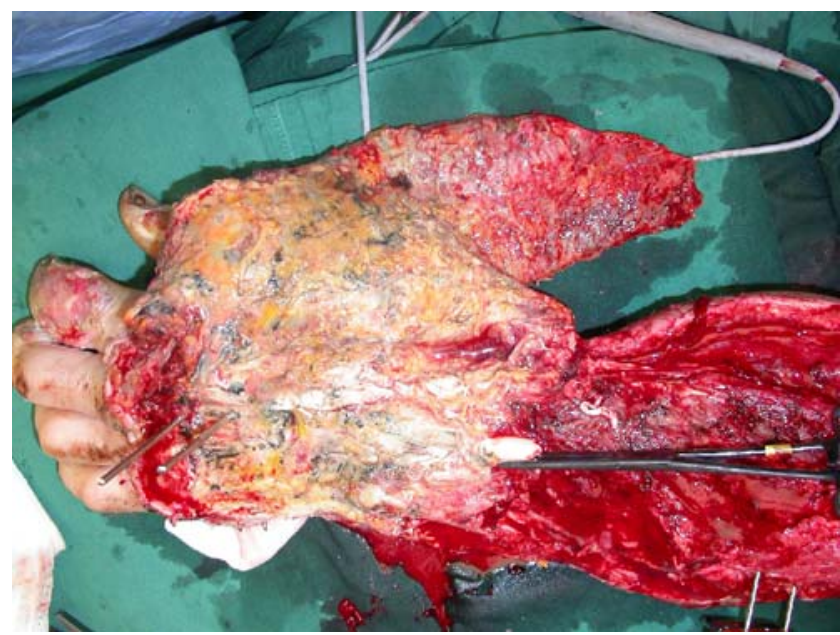

Fig. 6 Spreading of the fungal infection (perioperative view)

Successful use of this agent has already been described as a treatment of patients with Mucormycosis infection which was unresponsive to systemic administration of amphotericin B [12]. Ten days after the reconstruction there was a rapid progression and deterioration of the infection; wound exploration showed fungal contamination and discharge engulfing the entire wounds (Fig. 6). There was obvious fungal infection within the radius and ulna. The flap was viable, though surrounded and invaded by fungus (Fig. 7). After multiple debridement efforts an above elbow amputation was performed as a life saving procedure.

\section{Discussion}

Microsurgical free tissue transfer to reconstruct the upper extremity allows repair of significant soft tissue defects. A wide variety of free flaps are available for reconstruction of the

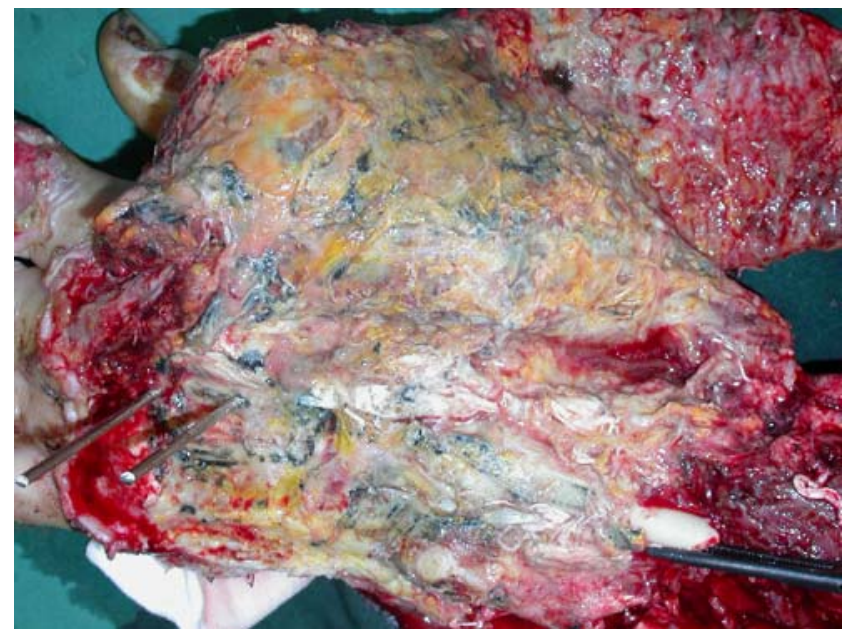

Fig. 7 Spreading of the fungal infection (closer view) 
large defects of this area. The size and location of the defect, and denuded structure are important factors, but the general status of the patient, his expectation, and the socioeconomic level should be considered in the planning of surgery [6].

Compound (chimeric) flaps can be used when different structures are necessary, such as vascularized bone, tendon or fascia; or when there is a need for filling up a defect.

A combined flap based on the chimeric concept consists of two different tissues, each having their own pedicle [6, 7].

Aggressive surgical debridement, following the concepts outlined by Hallock [5], should be performed before flap cover. Some organisms however fail to be eradicated by mechanical debridement as they are particularly pathogenic or embedded in the tissues.

In untidy wounds infected by Mucormycosis surgical management is almost always required, but the extent of debridement required for cure is often unclear. Few cases of surgical treatment of cutaneous mucormycosis are reported in the literature, and no uniform plan of treatment has been suggested. Currently, no clinical guidelines exist to assist the clinician in the surgical treatment of this potentially fatal disease.

Among all forms of mucormycosis, cutaneous mucormycosis is a relatively uncommon presentation and represents less than $10 \%$ of reported cases. It often occurs in patients who have sustained some form of insignificant superficial soft-tissue trauma, where it is supposed that the fungal spores, present in the skin, are deposited into the skin and deeper subcutaneous layers. The trauma need not be massive and may be as benign as an insect bite, intramuscular injection, or the use of contaminated elastic tape [9].

Primary cutaneous mucormycosis displays a broad spectrum of clinical disease, ranging from contamination to rapidly progressive gangrenous cellulitis, the latter usually indicating an immunocompromised host. Cutaneous mucormycosis is contracted either as a primary skin disease from direct inoculation, or secondarily via hematogenous metastasis from a pre-existing noncutaneous infection in another organ system. The secondary cutaneous form of mucormycosis is a manifestation of disseminated disease [9].

Cutaneous mucormycosis has been described by some as manifesting two clinical presentations: a subacute "superficial" form and a "gangrenous" form. If left untreated, this form of mucormycosis can reportedly disseminate hematogenously into the other, more severe form of the disease. "Gangrenous mucormycosis" has been described as the more severe form.

The mortality of Mucormycosis is $60 \%$ in patients treated only with systemic antimycotic agents, compared with $11 \%$ in patients who undergo surgical treatment [4]. A review of the literature yields a survival rate of only approximately 33\% after "radical surgical excision" or amputation and amphotericin B administration [12].
In the literature, clinically nonspecific language regarding surgical recommendations such as "conservative debridement", "radical surgical debridement", "wide excision", "radical surgery", "aggressive debridement", and "extensive surgical debridement" are used [2]. Reports in the literature suggest that although superficial mucormycosis can be treated with conservative surgical excision, gangrenous mucormycosis must be excised widely [9].

From a surgical standpoint, the distinction between superficial and gangrenous forms of mucormycosis may be a spurious one. The combined surgical and medical antifungal chemotherapeutic approach to treatment remains the standard of care [9]. The classic school of thought conveying musculocutaneous flap coverage [3] for untidy and complex extremity soft tissue defects is being increasingly challenged. Comparable control of infection and stable wound coverage is currently achieved with perforator flaps and fasciocutaneous flaps [1]. Even after aggressive debridement and early soft tissue cover infection may lead to failure of the reconstruction procedure in some cases $[1,5,10]$. Refinements in microvascular surgery allow elegant and functionally effective options for limb reconstruction, but an adequate control of infection is the key point for the success of the treatment. A judicious radical debridement and effective antimicrobial or antifungal therapy is essential for limb salvage.

In this case, wide surgical debridement was performed before flap cover, a progressively disseminating fungal infection was encountered.

\section{References}

1. Calderon W, Chang N, Mathes SJ (1986) Comparison of the effect of bacterial inoculation in muscolocutaneous and fasciocutaneous flaps. Plast Reconstr Surg 77:785-794

2. Clark FL, Batra RS, Gladstone HB (2003) Mohs micrographic surgery is an alternative treatment method for cutaneous mucormycosis. Dermatol Surg 29:882-885

3. Derderian CA, Olivier WA, Baux G, Levine J, Gurtner GC (2003) Microvascular free-tissue transfer for traumatic defects of the upper extremity: a 25-year experience. J Reconstr Microsurg 19 (7):455-462

4. Eucker J, Sezer O, Graf B, Possinger K (2001) Mucormycoses. Mycoses 44:253-260

5. Hallock GG (1991) Complication of 100 consecutive local fasciocutaneous flaps. Plast Reconstr Surg 88:264-268

6. Hamdi M, Van Landuyt K, Blondeel P, Monstrey S (2004) Aesthetic and functional consideration in the reconstruction of large skin defects with free flaps. Clin Plast Surg 31:39-48

7. Hamdi M, Van Landuyt K, Monstrey S, Blondeel P (2004) A clinical experience with perforator flaps in the coverage of extensive defects of the upper extremity. Plast Reconstr Surg 113(4):1175-1183

8. Harii K, Torii S, Sekiguchi J (1978) The free lateral thoracic flap. Plast Reconstr Surg 62:212-222

9. Losee JE, Selber J, Vega S, Hall C, Scott G, Serletti JM (2002) Primary cutaneous Mucormycosis: guide to surgical management. Ann Plast Surg 49(4):385-390 
10. Pederson CW (2001) Upper extremity microsurgery. Plast Reconstr Surg 107:1524-1536

11. Sun QN, Najvar LK, Bocanegra R, Loebenberg D, Graybill JR (2002) In vivo activity of Posaconazole against Mucor spp. in an immunosuppressed-mouse model. AAC 46:2310 2312
12. Verma GR, Lobo DR, Walker R, Bose SM, Gupta KL (1995) Disseminated mucormycosis in healthy adults. J Postgrad Med 41 (2): $40-42$

13. Welsch MJ, Moon CM, Elston DM, Vogel P (2003) Invasive synergistic fungal infection after motor vehicle collision. Cutis 71:201-204 